\title{
Paracoccidioidomicose no Hospital Universitário de Brasília
}

\author{
Paracoccidioidomycosis at Brasilia's University Hospital
}

\author{
Maria Vitoria Silva Campos ${ }^{1}$, Gerson Oliveira Penna ${ }^{1}$, Cleudson Nery de Castro, \\ Mario Augusto Pinto de Moraes ${ }^{2}$, Marcelo Simão Ferreira ${ }^{3}$ \\ e João Barberino Santos ${ }^{1}$
}

\begin{abstract}
RESUMO
Foram estudados 76 pacientes com paracoccidioidomicose, assistidos no Hospital Universitário de Brasília, entre 1984 e 2005. 0 gênero masculino representou 82,9\% e a média de idade foi 42 anos. Atividades agropecuárias caracterizaram 54,9\% dos pacientes. Entre pacientes com a forma crônica, 87\% eram tabagistas e 55,3\% etilistas. Em 71 pacientes sem co-infecção por HIV/aids: a) houve recidiva da paracoccidioidomicose em 21 (29,6\%); b) a forma crônica ou mista acometeu 77,5\% dos pacientes, com predominância de comprometimento orofaríngeo (70,9\%) e pulmonar (67,3\%), além de lesões linfonodais (29,8\%), laríngeas (27,3\%) e cutâneas (16,4\%); c) na forma aguda/subaguda, predominou o comprometimento linfonodal $(81,3 \%)$, seguido por lesões cutâneas $(43,8 \%)$, resultando doença grave em $62,5 \%$ e moderada em 37,5\%. Cinco pacientes tinham coinfecção por HIV/aids, dos quais três tiveram a infecção fúngica disseminada associada a acentuada imunodepressão.
\end{abstract}

Palavras-chaves: Paracoccidioidomicose. Região Centro-Oeste. Distrito Federal.

\begin{abstract}
Seventy-six paracoccidioidomycosis patients attended at the university hospital of Brasília from 1984 to 2005 were studied. $82.9 \%$ were male and the mean age was 42 years. $54.9 \%$ of the patients were engaged in farming activities. Among the patients with the chronic form, $87 \%$ were smokers and 55.3\% consumed alcohol. Among 71 patients without HIV/AIDS coinfection: a) paracoccidioidomycosis was recurrent in 21 (29.6\%); b) the chronic or mixed form affected $77.5 \%$ of patients, predominantly in the oropharynx (70.9\%) and lungs $(67.3 \%)$, with lymph node lesions in $29.8 \%$, laryngeal lesions in $27.3 \%$ and cutaneous lesions in $16.4 \%$; c) in the acute/subacute form, lymph node lesions predominated (81.3\%), followed by cutaneous lesions in $43.8 \%$, which resulted in severe disease in $62.5 \%$ and moderate disease in $37.5 \%$. Five patients had HIV/AIDS coinfection and three of them presented disseminated fungal infection together with marked immunosuppression.
\end{abstract}

Key-words: Paracoccidioidomycosis. Center-West region. Federal District.

A paracoccidioidomicose (PCM) é a micose sistêmica mais importante da América Latina. 0 Brasil detém $80 \%$ dos casos da doença, com distribuição irregular, concentrando-se em áreas endêmicas nas regiões Sudeste, Sul e Centro-Oeste 81112152023 . No período entre 1980 e 1995, foi considerada a oitava causa de óbito por doenças infectoparasitárias crônicas e recorrentes no país ${ }^{6}$. Por não ser uma doença de notificação compulsória ${ }^{8}$, o número real de casos é subestimado, baseado em registros hospitalares, relatos na literatura e poucos dados oficiais.

Apesar de incluso na região endêmica do Centro-Oeste, não existem dados na literatura médica sobre a prevalência da PCM no Distrito Federal. O Hospital Universitário de Brasília (HUB) é um serviço de referência para doenças infecciosas e parasitárias, atraindo pacientes locais, do entorno e dos estados vizinhos. A amostra do HUB é representativa da prevalência e das características clínico-epidemiológicas da doença na região.

\section{PACIENTES E MÉTODOS}

Foram revisados os prontuários dos pacientes com diagnóstico confirmado de PCM, assistidos no HUB, entre 1984 e 2005. Os pacientes foram identificados utilizando-se das informações registradas nos setores: Enfermaria de Doenças Infecciosas e Parasitárias, Laboratório de Micologia, Centro de Anatomia Patológica, Departamento de Saúde Coletiva, e Farmácia.

\footnotetext{
1. Núcleo de Medicina Tropical, Universidade de Brasília, Brasília, DF. 2. Centro de Anatomia Patológica, Hospital Universitário de Brasília, Brasília, DF. 3. Instituto de Ciências Biomédicas, Departamento de Clínica Médica, da Universidade Federal de Uberlândia, Uberlândia, MG.

Endereço para correspondência: Drª Maria Vitoria Silva Campos. Núcleo de Medicina Tropical/UnB. Campus Universitário Darcy Ribeiro. Asa Norte. Caixa Postal 4517, 70904-970 Brasília, DF. Brasil.

Tel: 5561 3273-5008, Fax: 5561 3273-2811

e-mail: mavialex@yahoo.com.br

Recebido para publicação em: 01/06/2007

Aceito em: 07/03/2008
} 
A confirmação laboratorial baseou-se na positividade de pelo menos um dos exames micológico, histopatológico, ou citopatológico para Paracoccidioides brasiliensis. A classificação da apresentação clínica da PCM em formas aguda/subaguda ou crônica/mista seguiu os critérios descritos nos consensos sobre PCM $^{91621}$.

Coletou-se as informações clínicas e epidemiológicas registradas em cada prontuário. Não foi possível a caracterização dos hábitos etilismo e tabagismo, importantes no contexto da doença, porque raramente foram quantificados pelos examinadores.

Os dados foram registrados em um formulário padronizado e organizados em um banco de dados, utilizando-se o programa Epi-Info versão 6. Os cálculos estatísticos de análise descritiva, teste $\chi 2$ e teste de proporção foram realizados com o sistema SAS (Statistical Analysis System). Considerou-se o nível de significância quando o valor de $\mathrm{p}<0,05$.

\section{RESULTADOS}

0 número de casos de PCM, levantados no HUB entre 1984 e 2005, foi 112. Os casos foram confirmados mediante exame histopatológico, em 65 pacientes, com 58 (89,2\%) positivos; exame micológico direto em 47 pacientes, com $31(65,9 \%)$ positivos; cultura para fungos em 35 pacientes, com $23(65,7 \%)$ positivos; exame citopatológico em 11 pacientes, com 3 (27,3\%) positivos. A reação sorológica de imunodifusão foi realizada em 26 pacientes, com 21 (80,8\%) positivos; a reação intradérmica à paracoccidioidina em 9 pacientes, com $2(22,2 \%)$ positivos (Tabela 1).

Tabela 1 - Freqüência dos métodos utilizados para o diagnóstico da paracoccidioidomicose.

\begin{tabular}{lcrrr}
\hline Métodos & Pacientes & \multicolumn{2}{c}{ Positividade } \\
\cline { 3 - 5 } & $\mathbf{n}^{\mathbf{0}}$ & & $\mathbf{n}^{\mathbf{0}}$ & $\mathbf{\%}$ \\
\hline Histopatológico & 65 & 58 & 89,2 \\
Micológico direto & 47 & 31 & 65,9 \\
Cultura & 35 & 23 & 65,7 \\
Citopatológico & 11 & 3 & 27,3 \\
Exame sorológico & 26 & 21 & 80,8 \\
Intradermorreação & 9 & 2 & 22,2 \\
\hline
\end{tabular}

Foram excluídos do estudo 33 pacientes com diagnóstico micológico ou histopatológico de Paracoccidioides brasiliensis positivo, sem registro de dados clínicos, e outros três pacientes cujo diagnóstico clínico não teve confirmação laboratorial. Desta forma, a amostra foi constituída de 76 pacientes.

Em 74 pacientes que informaram a naturalidade, 33 (44,6\%) eram do Nordeste e $23(31,1 \%)$ do Centro-Oeste, entre os quais $6(8,1 \%)$ do Distrito Federal. Quanto ao local de procedência, informado por 75 pacientes, a maioria $54(72 \%)$ provinha do Centro-Oeste, incluindo 32 (42,7\%) procedentes do Distrito Federal. Entre 65 pacientes, 36 (55,4\%) eram pardos, 21 (32,3\%) brancos e, 8 (12,3\%) negros. A atividade ocupacional de 71 pacientes foi distribuída em agropecuária 39 (54,9\%), sem significância estatística $(\mathrm{p}=0,4913)$; construção civil 6 (8,4\%) e garimpo $2(2,8 \%) ; 2$ (2,8\%) eram crianças. Dos pacientes com a forma crônica, tabagismo foi relatado por 47 (87\%) e etilismo por $26(55,3 \%)$.

A média de idade foi 42 anos, desvio padrão 15,25 e mediana 43,5 (6-77) anos nos 76 pacientes (Tabela 2). 0 tempo de doença até o estabelecimento diagnóstico, em 70 pacientes, teve média de 12 meses, desvio padrão 17,26 e mediana 6 (1-108) meses.

Desde a primeira notificação de infecção por HIV/aids no HUB, em 1989, foram assistidos 625 pacientes até o ano de 2005. A co-infecção PCM/HIV/aids foi relatada em 5 (6,6\%) dos 76 pacientes estudados (Tabela 3). A incidência da co-infecção PCM/HIV/aids, na população com HIV/aids assistida, foi 0,8\%. Os resultados expostos a seguir se referem aos 71 pacientes sem coinfecção por HIV/aids (dados específicos dos casos co-infectados serão apresentados em outro artigo).

Tabela 2 - Distribuição dos pacientes com paracoccidioidomicose, por faixa etária.

\begin{tabular}{lcc}
\hline Faixa etária (anos) & Número & Percentual \\
\hline 6 a 9 & 1 & 1,3 \\
10 a 19 & 5 & 6,6 \\
20 a 29 & 11 & 14,5 \\
30 a 39 & 16 & 21,0 \\
40 a 49 & 16 & 21,0 \\
50 a 59 & 18 & 23,7 \\
60 a 69 & 7 & 9,2 \\
70 a 79 & 2 & 2,6 \\
\hline Total & 76 & $\mathbf{1 0 0 , 0}$ \\
\hline
\end{tabular}

Tabela 3 - Gênero, forma clínica e co-infecção paracoccidioidomicose/ HIV/aids.

\begin{tabular}{|c|c|c|c|c|c|c|}
\hline \multirow[t]{2}{*}{ Forma clínica } & \multicolumn{2}{|c|}{ Masculino } & \multicolumn{2}{|c|}{ Feminino } & \multicolumn{2}{|c|}{ Total } \\
\hline & $n^{0}$ & $\%$ & $n^{0}$ & $\%$ & $\mathrm{n}^{\mathbf{0}}$ & $\%$ \\
\hline Sem co-infecção & 59 & 53,1 & 12 & 16,9 & 71 & 100,0 \\
\hline Forma aguda ou subaguda & 13 & 81,3 & 3 & 18,7 & 16 & 22,6 \\
\hline Forma crônica & 46 & 83,6 & 9 & 16,4 & 55 & 77,4 \\
\hline Com co-infecção & 4 & 80,0 & 1 & 20,0 & 5 & 100,0 \\
\hline Forma aguda ou subaguda & 2 & 66,7 & 1 & 33,3 & 3 & 60,0 \\
\hline Forma crônica ou mista & 2 & 100,0 & - & - & 2 & 40,0 \\
\hline Total & 63 & 82,9 & 13 & 17,1 & 76 & 100,0 \\
\hline
\end{tabular}

0 gênero masculino representou $59(93,6 \%)$ pacientes. Houve duas ocorrências pediátricas: um menino com 6 anos de idade apresentou forma disseminada, com caquexia, febre, hepatoesplenomegalia, nódulos subcutâneos, lesões osteoarticulares difusas, com óbito; uma adolescente de 13 anos com hepatoesplenomegalia, linfadenomegalia superficial e mesentérica difusas, permanece em acompanhamento. Apenas um caso foi confirmado em gestante.

A forma crônica da PCM ocorreu em 55 (77,5\%) e a forma aguda/subaguda em 16 (22,5\%). Nas três primeiras décadas de vida, a forma aguda/subaguda foi exclusiva, enquanto que, na quarta década, abrangeu apenas $2(15,4 \%)$ pacientes. Nos pacientes com a forma crônica (55), foi freqüente 0 comprometimento de orofaringe $39(70,9 \%)$ e dos pulmões 
37 (67,3\%). As lesões laríngeas 15 (27,3\%) foram exclusivas da forma crônica. Lesões linfonodais foram vistas em 17 (29,8\%) pacientes com a forma crônica e em 13 (81,3\%) com a forma aguda/subaguda e, as lesões cutâneas, em 9 (16,4\%) com a forma crônica e em 7 (43,8\%) com a forma aguda/subaguda. Na Tabela 4, pode-se observar, em resumo, as apresentações clínicas da forma aguda/subaguda da PCM.

A evolução foi grave em 4 (7,7\%), moderada em 30 (54,5\%) e leve em 21 (38,2\%) pacientes com a forma crônica. Na forma aguda/ subaguda, foi grave em 10 (62,5\%) e moderada em 6 (37,5\%). A recidiva clínica foi observada em 21 (29,6\%) pacientes.

Entre os critérios definidores de gravidade ${ }^{16}$, comprometimento intra-abdominal foi diagnosticado em 12 pacientes (oito com a forma aguda/subaguda e quatro com a forma crônica); e linfadenomegalia nodular mediastinal, em três.

Tabela 4 - Sinopse da apresentação clínica dos pacientes com a forma aguda/subaguda da paracoccidioidomicose.

\begin{tabular}{|c|c|c|}
\hline Gênero & $\begin{array}{l}\text { Idade } \\
\text { (anos) }\end{array}$ & Apresentação \\
\hline$\pi$ & 20 & linfadenomegalia cervical concomitante a tuberculose intestinal \\
\hline $\bar{q}$ & 23 & linfadenomegalia periférica e mediastinal \\
\hline $\bar{\sigma}$ & 24 & lesões cutâneas faciais \\
\hline$\delta$ & 23 & lesão íleocolônica e linfadenomegalia intra-abdominal \\
\hline$\pi$ & 18 & lesão cutânea única em coxa \\
\hline$\pi$ & 19 & $\begin{array}{l}\text { linfadenomegalia periférica, gomas em tórax, co-infecção com } \\
\text { leishmaniose tegumentar americana }\end{array}$ \\
\hline $\bar{\partial}$ & 26 & $\begin{array}{l}\text { linfadenomegalia intra-abdominal e periférica, ascite recidivante, } \\
\text { lesões cutâneas }\end{array}$ \\
\hline q & 30 & hepatoesplenomegalia e anemia (gestante) \\
\hline$\pi$ & 17 & linfadenomegalia periférica difusa \\
\hline$\pi$ & 29 & linfadenomegalia periférica e intra-abdominal, hepatomegalia \\
\hline$\pi$ & 30 & abscesso intra-abdominal, insuficiência renal crônica \\
\hline$\overline{0}$ & 20 & $\begin{array}{l}\text { quadro consuntivo, linfadenopatia periférica difusa e intra- } \\
\text { abdominal, hepatoesplenomegalia, derrame pleural refratário }\end{array}$ \\
\hline 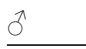 & 21 & linfadenomegalia hilar e periférica, lesões cutâneas disseminadas \\
\hline$\pi$ & 6 & lesões ósteoarticulares e cutâneas difusas, esplenomegalia \\
\hline$\pi$ & 19 & colangite de repetição e dilatação cística do colédoco \\
\hline $\bar{o}$ & 13 & linfadenomegalia mesentérica e periférica \\
\hline
\end{tabular}

Tuberculose foi a co-morbidade mais freqüente (Tabela 5). Um paciente desenvolveu doença de Addison 15 anos após o diagnóstico de PCM em orofaringe. Cinco (6,5\%) pacientes desenvolveram neoplasia maligna na vigência da micose: carcinoma basocelular

Tabela 5 - Freqüiência de co-morbidades em pacientes sem a co-infecção por HIV/aids.

\begin{tabular}{lc}
\hline Co-morbidades & Número \\
\hline Tuberculose & 5 \\
Doença de Chagas & 4 \\
Pitiríase versicolor & 2 \\
Ancilostomíase & 2 \\
Leishmaniose tegumentar americana & 1 \\
Estrongiloidíase & 1 \\
Amebíase intestinal & 1 \\
Sífilis & 1 \\
Brucelose & 1 \\
\hline
\end{tabular}

em face (2), carcinoma em pulmão e próstata (1), carcinoma epidermóide em olho (1) e, carcinoma epidermóide na boca, metastático para linfonodo cervical e fígado (1).

As drogas mais utilizadas, de forma isolada ou em associação seqüencial, para o tratamento de 71 pacientes (em 5 pacientes o diagnóstico ainda não era conhecido) foram: sulfametoxazoltrimetoprim (SMT-TMP) em 53 (74,6\%), anfotericina B em 16 (22,5\%), itraconazol em 15 (21,1\%), cetoconazol em 13 (18,3\%) e sulfadiazina em $9(12,7 \%)$. A monoterapia foi empregada em 41 (57,7\%) pacientes; a associação de duas drogas em 21 $(29,6 \%)$, de três drogas em $7(9,9 \%)$ e de quatro drogas em $2(2,8 \%)$.

Houve seis óbitos, entre os quais três de pacientes com a coinfecção PCM/HIV/aids. 0 tempo de seguimento hospitalar foi em média 394,33 dias $(\mathrm{DP}=349,18)$ e a mediana 355,5 dias (3-844).

\section{DISCUSSÃO}

O Distrito Federal localiza-se em região considerada de moderada a elevada endemicidade de paracoccidioidomicose ${ }^{12620}$. Casos autóctones foram suspeitados em seis pacientes jovens residentes no Distrito Federal, sem história de viagens para outros estados, um deles co-infectado por HIV. Apesar da PCM ter um aspecto ocupacional ligado às atividades agropecuárias ${ }^{51121523}$, pode ocorrer em pessoas que sempre residiram em áreas urbanas ${ }^{51324}$.

A paracoccidioidomicose prevaleceu no gênero masculino, entre a $4^{\mathrm{a}}$ e a $6^{\mathrm{a}}$ décadas de vida ${ }^{511} 1521$. A proporção encontrada entre os gêneros masculino e feminino $(4,8: 1)$ varia conforme a região, sendo 4:1 em Goiás e 125:1 no Rio Grande do Sul2 271520 .

A grande frequiência de casos moderados e graves justifica-se em amostra de um hospital terciário, referência para doenças infecciosas e parasitárias.

A baixa prevalência de comprometimento respiratório em relação a outros estudos ${ }^{112023}$ sugere que a hipótese de micose pulmonar tenha sido descartada pelo valor limitado da radiografia simples de tórax na avaliação de doenças pulmonares difusas. A investigação de micose pulmonar deve ser complementada com tomografia computadorizada ${ }^{17}$, exame seriado de escarro e/ou broncoscopia com lavado broncoalveolar ${ }^{10}$, os quais foram raramente realizados.

A elevada correlação entre hábitos de tabagismo e etilismo e comprometimento respiratório crônico ${ }^{2024}$, inclusive com lesões laríngeas ${ }^{402425}$, foi condizente com a literatura.

0 exame histopatológico continua sendo o pilar para 0 diagnóstico da $\mathrm{PCM}^{13}$, devido à sua sensibilidade. Contudo, em alguns pacientes, o diagnóstico da PCM só foi possível através da cultura para fungos. Outros métodos diagnósticos foram úteis, porém menos empregados.

O largo uso de SMT-TMP ${ }^{19} 2023$ se deve à eficácia alcançada contra as formas leves e moderadas e à disponibilidade do medicamento na rede pública de saúde. 0 itraconazol foi usado em apenas $21,1 \%$, devido ao seu alto custo, embora a tendência atual seja recomendá-lo como primeira opção contra formas 
leves e moderadas ${ }^{21}$. Alguns pacientes foram tratados com 0 cetoconazol, embora atualmente não seja mais recomendado para o tratamento. A anfotericina B continua sendo a droga de eleição para as formas graves e para as falhas de tratamento $0^{132123}$.

Não foi possível esclarecer se a doença de Addison desenvolvida em um paciente após a introdução da terapia antimicótica, foi de origem auto-imune, ou devida à adrenalite granulomatosa causada pela $\mathrm{PCM}^{22}$.

A taxa de co-infecção PCM/HIV/aids foi pequena ${ }^{31418}$, talvez por ter sido pouco investigada, uma vez que a padronização de rotinas para suspeição e diagnóstico de HIV/aids só ocorreu após 1984. Os cinco pacientes co-infectados habitavam área urbana. A co-infecção PCM/HIV/aids é incomum quando comparada a outras micoses superficiais e sistêmicas ${ }^{10}$. Mesmo assim, a vigilância diagnóstica desta associação, mormente em pacientes do campo, deve ser constante e os serviços de referência estimulados a fazê-la.

\section{REFERÊNCIAS}

1. Andrade ALSS. Paracoccidioidomicose linfático-abdominal - contribuição ao seu estudo. Revista Patologia Tropical 12: 165-256, 1983.

2. Barbosa W. Blastomicose sul-americana. Contribuição ao seu estudo no Estado de Goiás. Tese de Livre-Docência, Universidade Federal de Goiás, Goiânia, G0, 1968.

3. Bernard G, Duarte AJS. Paracoccidioidomycosis: a model for evaluation of the effects of human immunodeficiency virus infection on the natural history of endemic tropical diseases. Clinical Infectious Diseases 31: 1032-1039, 2000.

4. Bisinelli JC, Telles FQ, Sobrinho JA, Rapoport A. Manifestações estomatológicas da paracoccidioidomicose. Revista Brasileira de Otorrinolaringologia 67: 683-687, 2001.

5. Blotta MHSL, Mamoni RL, Oliveira SJ, Nouér SA, Papaiordanou PMO, Goveia A, Camargo ZP. Endemic regions of paracoccidioidomycosis in Brazil: a clinical and epidemiologic study of 584 cases in the Southeast region. The American Journal of Tropical Medicine and Hygiene 61: 390-394, 1999.

6. Coutinho ZF, Silva D, Lazéra M, Petri V, Oliveira RM, Sabroza PC, Wanke B. Mortalidade por paracoccidioidomicose no Brasil (1980-1995). Cadernos de Saúde Pública 18: 1441-1454, 2002.

7. Ferreira MS. Contribuição para o estudo clínico-laboratorial e terapêutico da forma juvenil da paracoccidioidomicose. Tese de Livre-Docência, Universidade Estadual do Rio de Janeiro, Rio de Janeiro, RJ, 1988.

8. Fornajeiro N, Maluf MLF, Takahachi G, Svidzinski. Inquérito epidemiológico sobre a paracoccidioidomicose utilizando a gp 43 em dois municípios do noroeste do Paraná, Brasil. Revista da Sociedade Brasileira de Medicina Tropical 38:191-193, 2005

9. Franco M, Montenegro MR, Mendes RP, Marques SA, Dillon NL, Mota NGS. Paracoccidioidomycosis: a recently proposed classification of its clinical forms. Revista da Sociedade Brasileira de Medicina Tropical 20: 129-132, 1987.
10. Lazzarini-de-Oliveira LC, Arantes AA, Caiuby MJM. Utilidade da investigação rotineira de infecção fúngica pela broncoscopia em pacientes infectados ou não pelo HIV em um hospital geral, referência para SIDA. Revista da Sociedade Brasileira de Medicina Tropical 32: 255-261, 1999.

11. Londero AT, Ramos CD. Paracoccidioidomicose: estudo clínico-micológico de 260 casos observados no interior do Estado do Rio Grande do Sul. Jornal de Pneumologia 16: 129-132, 1990.

12. Maluf MLF, Pereira SRC, Takahachi G, Svidzinski TIE. Prevalência da paracoccidioidomicose-infecção determinada através de teste sorológico em doadores de sangue na região Noroeste do Paraná, Brasil. Revista da Sociedade Brasileira de Medicina Tropical 36: 11-16, 2003.

13. Marques SA. Paracoccidioidomicose: atualização epidemiológica, clínica e terapêutica. Anais Brasileiros de Dermatologia 78: 135-150, 2003.

14. Marques SA, Conterno LO, Sgarbi LP, Villagra AMPC, Sabongi VPG, Bagatin E, Gonçalves VLC. Paracoccidioidomycosis associated with acquired immunodeficiency syndrome. Report of seven cases. Revista do Instituto de Medicina Tropical de São Paulo 37: 261-265, 1995.

15. Marques SA, Franco MF, Mendes RP, Silva NCA, Baccili C, Curcelli ED. Aspectos epidemiológicos da paracoccidioidomicose na área endêmica de Botucatu (São Paulo - Brasil). Revista do Instituto de Medicina Tropical de São Paulo 25: 87-92, 1983

16. Mendes RP. Paracoccidioidomicose. In: Cimerman S, Cimerman B. Condutas em infectologia. 1a edição, Editora Atheneu, São Paulo, p. 384-399, 2004.

17. Muniz MAS, Marchiori E, Magnago M, Moreira LBM, Almeida Junior JG Paracoccidioidomicose pulmonar - Aspectos na tomografia computadorizada de alta resolução. Radiologia Brasileira 35:147-154, 2002.

18. Nobre V, Braga E, Rayes A, Serufo JC, Godoy P, Nunes N, Antunes CM, Lambertucci JR. Opportunistic infections in patients with AIDS admitted to an university hospital of the South of Brazil. Revista do Instituto de Medicina Tropical de São Paulo 45: 69-74, 2003

19. Nogueira MGS, Andrade GMQ, Tonelli E, Diniz SN, Goes AM, Cisalpino OS. Aspectos laboratoriais evolutivos de crianças em tratamento da paracoccidioidomicose. Revista da Sociedade Brasileira de Medicina Tropical 39: 478-483, 2006.

20. Paniago AMM, Aguiar JIA, Aguiar ES, Cunha RV, Pereira GROL, Londero AT, Bodo W. Paracoccidioidomicose: estudo clínico e epidemiológico de 422 casos observados no Estado de Mato Grosso do Sul. Revista da Sociedade Brasileira de Medicina Tropical 36: 455-459, 2003.

21. Shikanai-Yasuda MA, Telles Filho FQ, Mendes RP, Colombo AL, Moretti ML. Consenso em paracoccidioidomicose. Revista da Sociedade Brasileira de Medicina Tropical 39: 297-310, 2006.

22. Silva RC, Castro M, Kater CE, Cunha AA, Moraes AM, Alvarenga DB, Moreira AC, Elias LLK. Insuficiência Adrenal Primária no Adulto: 150 Anos Depois de Addison. Arquivos Brasileiros de Endocrinolologia e Metabologia 48: 724-738, 2004.

23. Valle ACF. Tratamento da paracoccidioidomicose: estudo retrospectivo de 500 casos. Análise clínica, laboratorial e epidemiológica. Anais Brasileiros de Dermatologia 67: 251-254, 1992.

24. Verli FD, Marinho SA, Souza SC, Figueiredo MAZ, Yurgel LS. Perfil clínicoepidemiológico dos pacientes portadores de paracoccidioidomicose no Serviço de Estomatologia do Hospital São Lucas da Pontifícia Universidade Católica de Rio Grande do Sul. Revista da Sociedade Brasileira de Medicina Tropical 38: 234-237, 2005.

25. Vieira EMM, Borsatto-Galera B. Manifestações clínicas bucais da paracoccidioidomicose. Revista de Patologia Tropical 35: 23-30, 2006. 